\title{
Strain gradient theory with couple stress for crystalline solids
}

\author{
Shaohua Chen*, Tzuchiang Wang \\ LNM, Institute of Mechanics, Chinese Academy of Sciences, Beijing, 100080, China
}

(Received 20 November 2000; revised and accepted 16 May 2001)

\begin{abstract}
A new phenomenological strain gradient theory for crystalline solid is proposed. It fits within the framework of general couple stress theory and involves a single material length scale $l_{c s}$. In the present theory three rotational degrees of freedom $\omega_{i}$ are introduced, which denote part of the material angular displacement $\theta_{i}$ and are induced accompanying the plastic deformation. $\omega_{i}$ has no direct dependence upon $u_{i}$ while $\boldsymbol{\theta}=(1 / 2)$ curl $\mathbf{u}$. The strain energy density $w$ is assumed to consist of two parts: one is a function of the strain tensor $\varepsilon_{i j}$ and the curvature tensor $\chi_{i j}$, where $\chi_{i j} \equiv \omega_{i, j}$; the other is a function of the relative rotation tensor $\alpha_{i j} . \alpha_{i j}=e_{i j k}\left(\omega_{k}-\theta_{k}\right)$ plays the role of elastic rotation tensor. The anti-symmetric part of Cauchy stress $\tau_{i j}$ is only the function of $\alpha_{i j}$ and $\alpha_{i j}$ has no effect on the symmetric part of Cauchy stress $\sigma_{i j}$ and the couple stress $m_{i j}$. A minimum potential principle is developed for the strain gradient deformation theory. In the limit of vanishing $l_{c s}$, it reduces to the conventional counterparts: $J_{2}$ deformation theory. Equilibrium equations, constitutive relations and boundary conditions are given in detail. For simplicity, the elastic relation between the anti-symmetric part of Cauchy stress $\tau_{i j}$, and $\alpha_{i j}$ is established and only one elastic constant exists between the two tensors. Combining the same hardening law as that used in previously by other groups, the present theory is used to investigate two typical examples, i.e., thin metallic wire torsion and ultra-thin metallic beam bend, the analytical results agree well with the experiment results. While considering the stretching gradient, a new hardening law is presented and used to analyze the two typical problems. The flow theory version of the present theory is also given. (C) 2001 Éditions scientifiques et médicales Elsevier SAS
\end{abstract}

strain gradient / crystalline solids / couple stress / hardening law

\section{Introduction}

There is accumulating experimental evidence for the existence of material size effects in plasticity, with the feature that the smaller the imposed geometric length scale relative to some material length scales, the stronger the material in its plastic response. In the experiments of measuring micro-indentation hardness of metallic materials the square of the hardness increases linearly as the depth of indentation decreases (Nix, 1989; Ma and Clarke, 1995; Poole et al., 1996; McElhaney et al., 1998). In reinforced metal matrix composites, small particles give rise to an enhanced rate of strain hardening compared to the same volume fraction of larger particles (Kelly and Nicholson, 1963; Ebeling and Ashby, 1966; Lloyd, 1994). The overall properties of a polycrystal are functions of $l / a$, which reflects the grain size effect, where $l$ is the intrinsic length scale introduced into crystal's constitutive law by the gradient effects and $a$ is the grain size (Smyshlyaev and Fleck, 1996). The most compelling experimental evidence that strong size effects exist have been provided by Fleck et al. (1994) and Stolken and Evans (1998). The former is to measure the scaled shear strength while twisting thin wires with different diameters and the latter is to measure the bend moments while bending ultra-thin beams with different thickness.

In recent years several theories (Dillon et al. (1970); Fleck and Hutchinson (1993); Fleck et al. (1994, 1997); Gao et al. (1999)) on strain gradient effects have been proposed. Among them, Fleck and Hutchinson (1993)

\footnotetext{
* Correspondence and reprints.

E-mail address: chenshaohua72@hotmail.com (S. Chen).
} 
and Fleck et al. (1994) developed a phenomenological strain gradient theory of plasticity, which models the hardening due to both statistically stored and geometrically necessary dislocations. Their theory fits neatly within the general framework of the reduced couple stress theory. There are a lot of works about couple stress theory, such as those of Toupin (1962), Mindlin (1963, 1964), Schaefer (1967) and Eringen (1968). Green, Mcinnis and Naghdi (1968) proposed a dipolar theory of plasticity in the presence of simple force and stress dipoles. Naghdi and Srinivasa (1993-a, 1993-b, 1994) developed a Cosserat theory with three directors and solved problems involving the evolution of dislocations.

In contrast, no work conjugate of strain gradient has been defined in the alternative gradient theories (Aifantis, 1984; Muhlhaus and Aifantis, 1991). Retaining the essential structure of conventional plasticity and obeying thermodynamic restrictions, Acharya and Bassani (1995) concludes that the only possible formulation is a flow theory with strain gradient effects represented as an internal variable, which acts to increase the current tangent-hardening modulus. However, there has not been a systematic way of constructing the tangent modulus so as to validate this framework.

Shizawa and Zbib (1999) developed a thermodynamic theory of gradient elastoplasticity by introducing the concept of the dislocation density tensor.

Chen and Wang (2000) established a new hardening law based on the incremental version of conventional $J_{2}$ deformation theory, in which the effective strain gradient is only a parameter to influence the tangent modulus.

In the present paper, a new phenomenological strain gradient theory for crystalline solid is proposed based on the general couple stress theory. Independent variable $\omega_{i}$, which is the angular displacement accompanying plastic deformation is introduced. Using the same hardening law as that in Fleck et al. (1994), thin metallic wire torsion and ultra-thin metallic beam bend, are investigated. Furthermore, a new hardening law including the stretch gradient is proposed to analyze the same two problems. In the current paper, finite strain effects are neglected: no distinction is made between the initial undeformed configuration and the current deformed configuration.

A brief review on general couple stress theory is given in Section 2. A new strain gradient deformation theory is given in Section 3. In Section 4, the problem of thin wire torsion is analyzed using the new strain gradient deformation theory with the same hardening law as that proposed by Fleck and Hutchinson (1993) and ultra-thin beam bend is analyzed in Section 5. A new hardening law including the stretch gradient is given in Section 6. The corresponding flow theory version for the new theory is given in Section 7.

\section{Review of the general couple stress theory}

In the general couple stress theory (Mindlin, 1963, 1964), $\omega$ is called micro-rotation and is treated as an independent kinematic quantity with no direct dependence upon $\mathbf{u}$ and distinct from the material rotation, $\boldsymbol{\theta} \equiv(1 / 2)$ curl $\mathbf{u}$. $\boldsymbol{\sigma}$ denotes the symmetric part of the Cauchy stress and $\tau$ denotes the anti-symmetric part of the Cauchy stress, $\mathbf{m}$ denotes the couple stress tensor.

Neglecting the body forces and body couples, the equilibrium relation of forces within body gives:

$$
t_{i j, j}=\sigma_{i j, j}+\tau_{i j, j}=0,
$$

and moment equilibrium implies:

$$
\tau_{j k}=\frac{1}{2} e_{i j k} m_{i p, p} .
$$


Here, a comma indicates a partial derivative with respect to a Cartesian coordinate and a repeated suffix denotes summation over 1 to 3 . A subscript index can take the value of 1,2 or 3 and $e_{i j k}$ denotes the usual permutation symbol.

Traction equilibrium on the surface $S$ of the body is:

$$
T_{i}=\left(\sigma_{i j}+\tau_{i j}\right) n_{j}, \quad q_{i}=m_{i j} n_{j},
$$

where $T_{i}$ and $q_{i}$ are the surface traction and surface torque per unit area; $n_{i}$ is the $i$ th component of the unit normal vector $\mathbf{n}$ at the surface of the body.

The principle of virtual work for the general couple stress theory is:

$$
\int_{V}\left[\left(\sigma_{i j}+\tau_{i j}\right) \delta \gamma_{i j}+m_{i j} \delta \chi_{i j}\right] \mathrm{d} V=\int_{S}\left[T_{i} \delta u_{i}+q_{i} \delta \omega_{i}\right] \mathrm{d} S
$$

where $\chi_{i j}=\omega_{i, j}$ is the micro-curvature tensor and $\gamma_{i j}=u_{i, j}+e_{i j k} \omega_{k}$ is called the relative displacement gradient tensor.

The above virtual work statement can be rearranged to the form:

$$
\int_{V}\left[\sigma_{i j} \delta \varepsilon_{i j}+\tau_{i j} \delta \alpha_{i j}+m_{i j} \delta \chi_{i j}\right] \mathrm{d} V=\int_{S}\left[T_{i} \delta u_{i}+q_{i} \delta \omega_{i}\right] \mathrm{d} S,
$$

where the symmetric tensor $\varepsilon_{i j}$ is the conventional strain tensor:

$$
\varepsilon_{i j}=\left(u_{i, j}+u_{j, i}\right) / 2,
$$

and the anti-symmetric tensor $\alpha_{i j}$ is the relative rotation tensor:

$$
\alpha_{i j}=e_{i j k} \omega_{k}-\left(u_{j, i}-u_{i, j}\right) / 2=e_{i j k}\left(\omega_{k}-\theta_{k}\right) .
$$

The strain energy density function $w$ in the general couple stress theory depends upon the strain tensor $\boldsymbol{\varepsilon}$, the curvature tensor $\chi$ and the relative rotation $\alpha$ according to:

$$
w(\boldsymbol{\varepsilon}, \boldsymbol{\chi}, \boldsymbol{\alpha})=\int_{0}^{\varepsilon_{i j}} \sigma_{i j} \mathrm{~d} \varepsilon_{i j}+\int_{0}^{\chi_{i j}} m_{i j} \mathrm{~d} \chi_{i j}+\int_{0}^{\alpha_{i j}} \tau_{i j} \mathrm{~d} \alpha_{i j} .
$$

Then, the constitutive relations are:

$$
\sigma_{i j}=\frac{\partial w}{\partial \varepsilon_{i j}}, \quad m_{i j}=\frac{\partial w}{\partial \chi_{i j}}, \quad \tau_{i j}=\frac{\partial w}{\partial \alpha_{i j}} .
$$

\section{Strain gradient deformation theory}

As we know the gradient of displacement $\beta_{i j}$ can be written as:

$$
\beta_{i j}=\partial_{i} u_{j}
$$

and it can be divided into two parts: strain tensor and rotation tensor:

$$
\beta_{i j}=\varepsilon_{i j}-W_{i j}
$$


For crystalline solid, the rotation tensor $W_{i j}$ can be decomposed into two parts:

$$
W_{i j}=W_{i j}^{e}+W_{i j}^{p}
$$

where $W_{i j}^{e}$ is the elastic rotation tensor and $W_{i j}^{p}$ is the plastic rotation tensor.

The angular displacement accompanying the plastic deformation can be chosen as an independent rotation vector $\omega_{i}$, which is directly related to the plastic rotation tensor $W_{i j}^{p}$ according to the following formula:

$$
W_{i j}^{p}=-e_{i j k} \omega_{k}
$$

The rotation vector $\theta_{i}$ corresponding to $W_{i j}$ denotes the material angular displacement, $\boldsymbol{\theta} \equiv(1 / 2)$ curl $\mathbf{u}$. The relative curvature tensor is $\alpha_{i j}=e_{i j k}\left(\omega_{k}-\theta_{k}\right)=W_{i j}^{e}$, which is an anti-symmetric tensor and directly relates to the rotation vector accompanying elastic deformation. Since $\omega_{i}$ is independent and has no relation with the displacement vector $u_{i}, \alpha_{i j}$ does not vanish, which is different from the theory proposed by Fleck et al. (1994) and Fleck and Hutchinson (1993).

We define the symmetric part of Cauchy stress, $\sigma_{i j}$, as the work conjugate of the strain tensor $\varepsilon_{i j}$; the couple stress tensor $m_{i j}$ as the work conjugate of the curvature tensor $\chi_{i j}$, where $\chi_{i j}=\omega_{i, j}$. The anti-symmetric part of Cauchy stress $\tau_{i j}$, is the work conjugate of the relative curvature tensor $\alpha_{i j}$. The deviatoric part $s_{i j}$ of the Cauchy stress and the deviatoric part $m_{i j}^{\prime}$ of the couple stress are defined as the work conjugates of $\varepsilon_{i j}^{\prime}, \chi_{i j}^{\prime}$ respectively; $\sigma_{m}$ and $m_{m}$ are defined as the work conjugates of $\varepsilon_{m}$ and $\chi_{m}$ respectively, then one can obtain:

$$
\delta w=s_{i j} \delta \varepsilon_{i j}^{\prime}+m_{i j}^{\prime} \delta \chi_{i j}^{\prime}+\sigma_{m} \delta \varepsilon_{m}+m_{m} \delta \chi_{m}+\tau_{i j} \delta \alpha_{i j}
$$

where $s_{i j} \equiv \sigma_{i j}-(1 / 3) \delta_{i j} \sigma_{k k}, m_{i j}^{\prime} \equiv m_{i j}-(1 / 3) \delta_{i j} m_{k k}$ and the term $\tau_{i j} \delta \alpha_{i j}$ plays the role of the work produced by elastic curvature tensor.

Equation (14) enables one to determine $s_{i j}, m_{i j}^{\prime}, \sigma_{m}, m_{m}$ and $\tau_{i j}$ in terms of the strain and curvature tensor states of the solid as:

$$
s_{i j}=\frac{\partial w}{\partial \varepsilon_{i j}^{\prime}}, \quad m_{i j}^{\prime}=\frac{\partial w}{\partial \chi_{i j}^{\prime}}, \quad \sigma_{m}=\frac{\partial w}{\partial \varepsilon_{m}}, \quad m_{m}=\frac{\partial w}{\partial \chi_{m}}, \quad \tau_{i j}=\frac{\partial w}{\partial \alpha_{i j}} .
$$

In the present paper, we assume that the strain energy density $w$ can be expressed as:

$$
w(\boldsymbol{\varepsilon}, \boldsymbol{\chi}, \boldsymbol{\alpha})=w_{0}(\boldsymbol{\varepsilon}, \boldsymbol{\chi})+w_{1}(\boldsymbol{\alpha}) .
$$

It means that the anti-symmetric stress $\tau_{i j}$ depends only on the relative rotation tensor $\alpha_{i j}$. In other words, $\tau_{i j}$ is only the function of elastic rotation vector. Meanwhile the relative rotation tensor $\alpha_{i j}$ has no effect on $\sigma_{i j}$ and $m_{i j}$.

For isotropic material, $w_{1}$ should be the isotropic scalar function of the tensor $\alpha_{i j}$. Since $\alpha_{i j}$ is an antisymmetric tensor, according to Spencer (1971), $\alpha_{i j}$ has only one independent invariant, $\alpha_{i j} \alpha_{j i}$. Hence we have:

$$
w_{1}\left(\alpha_{i j}\right)=w_{1}\left(J_{2}\right),
$$

where

$$
J_{2}=\alpha_{i j} \alpha_{i j}
$$


Substituting equations (16) and (17) into (15), one can obtain:

$$
\sigma_{i j}=\frac{\partial w_{0}}{\partial \varepsilon_{i j}}, \quad m_{i j}=\frac{\partial w_{0}}{\partial \chi_{i j}}, \quad \tau_{i j}=\frac{\partial w_{1}}{\partial J_{2}} \cdot \frac{\partial J_{2}}{\partial \alpha_{i j}}=2 w_{1}^{\prime}\left(J_{2}\right) \alpha_{i j} .
$$

According to the work by Fleck and Hutchinson (1993) and Fleck et al. (1994), it is mathematically convenient to assume that the strain energy density $w_{0}$ depends only upon the single scalar strain measure $E_{e}$, where:

$$
E_{e}^{2}=\varepsilon_{e}^{2}+l_{c s}^{2} \chi_{e}^{2}
$$

The length scale $l_{c s}$ is a material length scale related with rotation gradient and required on dimensional grounds, $\varepsilon_{e}^{2}=\frac{2}{3} \varepsilon_{i j}^{\prime} \varepsilon_{i j}^{\prime}, \chi_{e}^{2}=\frac{2}{3} \chi_{i j}^{\prime} \chi_{i j}^{\prime}$.

An effective stress measure $\Sigma_{e}$ is defined as the work conjugate of $E_{e}$, which is proposed by Fleck and Hutchinson (1993) and the hardening relation between $\Sigma_{e}$ and $E_{e}$ is:

$$
\Sigma_{e}=\frac{\mathrm{d} w_{0}\left(E_{e}\right)}{\mathrm{d} E_{e}}=f\left(E_{e}\right)
$$

then

$$
s_{i j}=\frac{2 \Sigma_{e}}{3 E_{e}} \varepsilon_{i j}^{\prime}, \quad m_{i j}^{\prime}=\frac{2}{3} l_{c s}^{2} \frac{\Sigma_{e}}{E_{e}} \chi_{i j}^{\prime}, \quad \sigma_{m}=\frac{1}{3} \sigma_{k k}, \quad m_{m}=\frac{1}{3} m_{k k},
$$

and

$$
\Sigma_{e}=\left(\sigma_{e}^{2}+l_{c s}^{-2} m_{e}^{2}\right)^{1 / 2}
$$

where

$$
\begin{aligned}
\sigma_{e}^{2} & =\frac{3}{2} s_{i j} s_{i j}, & m_{e}^{2} & =\frac{3}{2} m_{i j}^{\prime} m_{i j}^{\prime}, \\
\varepsilon_{e}^{2} & =\frac{2}{3} \varepsilon_{i j}^{\prime} \varepsilon_{i j}^{\prime}, & \chi_{e}^{2} & =\frac{2}{3} \chi_{i j}^{\prime} \chi_{i j}^{\prime} .
\end{aligned}
$$

Consider a body of volume $V$ and surface $S$ comprised of non-linear elastic solid: the solid satisfies the constitutive law (19). Stress traction $T_{i}^{0}$ acts on a portion $S_{T}$ of the surface of the body, on the remaining portions $S_{u}$ the displacement is prescribed as $u_{i}^{0}$ and couple stress traction $q_{i}^{0}$ acts on a portion $S_{q}$, on the remaining portions $S_{\omega}$ of the surface the rotation is prescribed as $\omega_{i}^{0}$. Then the following principle of minimum potential energy may be stated:

Consider all admissible displacement fields $u_{i}$ and rotation vector fields $\omega_{i}$ which satisfy $u_{i}=u_{i}^{0}$ and $\omega_{i}=\omega_{i}^{0}$ on the part of the boundaries $S_{u}$ and $S_{\omega}$, respectively. The real displacement fields and the real rotation vector fields render the potential energy $P(\mathbf{u}, \boldsymbol{\omega})$ to be minimum. The potential energy $P(\mathbf{u}, \boldsymbol{\omega})$ is expressed as following:

$$
P(\mathbf{u}, \boldsymbol{\omega})=\int_{V} w(\boldsymbol{\varepsilon}, \boldsymbol{\chi}, \boldsymbol{\alpha}) \mathrm{d} V-\left(\int_{S_{T}} T_{i}^{0} u_{i} \mathrm{~d} S+\int_{S_{q}} q_{i}^{0} \omega_{i} \mathrm{~d} S\right) .
$$

The strain gradient theory proposed by Fleck and Hutchinson (1993) and Fleck et al. (1994) falls within the classification of reduced couple stress theory. The pertinent kinematic quantities in reduced couple stress theory are the displacement $\mathbf{u}$ and the overall rotation $\boldsymbol{\theta} \equiv(1 / 2)$ curl $\mathbf{u}$. The relative rotation tensor $\boldsymbol{\alpha}$ vanishes for the particular choice $\boldsymbol{\omega} \equiv \boldsymbol{\theta}$ and the curvature tensor $\chi_{i j}=\theta_{i, j}, \chi_{i i}=0$. The force equilibrium and moment 
equilibrium is the same as equations (1) and (2), respectively, but the moment $\mathbf{m}$ is only the deviatoric part and the spherical part $m_{m}$ can not enter the equilibrium equations.

In this paper, the rotation vector $\boldsymbol{\omega}$ is independent and has no relation with the displacement $\mathbf{u} . \chi_{i j}=\omega_{i, j}$ and its work conjugate, the couple stress $m_{i j}$, includes the deviatoric part $m_{i j}^{\prime}$ and the spherical part $m_{m}$. The spherical part of couple stress is not zero in the present paper since the independent rotation vector $\omega$ is introduced and different from other theories. The spherical part of couple stress plays an important role in obtaining the boundary conditions. Details about the importance of the spherical part's existence can be found in the paper by Green, Mcinnis and Naghdi (1968).

\section{Thin wire torsion}

As a typical example, we assume that:

$$
w_{1}=C_{1} J_{2}
$$

where $C_{1}$ is a material constant.

From equation (19), it follows:

$$
\tau_{i j}=2 C_{1} \alpha_{i j}
$$

In this section, torsion of thin copper wires with different diameters is analyzed using the present theory. A Cartesian coordinate system $\left(x_{1}, x_{2}, x_{3}\right)$ and a cylindrical polar coordinate system $\left(r, \theta, x_{3}\right)$ are introduced as shown in figure 1 and $x_{3}$ axis is parallel to the axis of the wire. The radius of the wire is $a . \kappa$ is the twist per unit length of the wire and taken to be positive without loss of generality.

The equilibrium equations for stresses and couple stresses are:

$$
\begin{aligned}
t_{i j, j} & =\sigma_{i j, j}+\tau_{i j, j}=0 ; \\
\tau_{j k} & =\frac{1}{2} e_{i j k} m_{i p, p} ; \\
\tau_{i j} & =2 C_{1} \alpha_{i j}=2 C_{1} e_{i j k}\left(\omega_{k}-\theta_{k}\right) .
\end{aligned}
$$

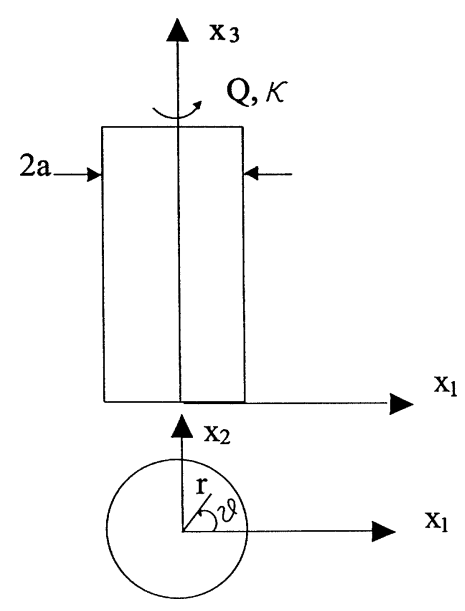

Figure 1. Coordinate systems $\left(x_{1}, x_{2}, x_{3}\right)$ and $\left(r, \theta, x_{3}\right)$ on a thin wire. 
Now we introduce $K_{1}$, which is called the volumetric modulus of bend-torsion, then we have:

$$
m_{m}=K_{1} l_{c s}^{2} \chi_{m},
$$

where $\chi_{m}=\frac{1}{3} \chi_{i i}$ and $m_{m}$ denotes the spherical part of couple stress, i.e. $m_{i j}=m_{i j}^{\prime}+m_{m} \delta_{i j}$.

In order to compare the results of the present theory and that proposed by Fleck and Hutchinson (1993) reasonably, the hardening relation, the traction boundary conditions and the displacement boundary conditions are taken to be the same as that used in Fleck et al. (1994). The solid is assumed to be incompressible for strain tensor.

The boundary conditions for couple stresses on the lateral face can be expressed as follows (details in Fleck et al. (1994)):

$$
\begin{gathered}
m_{\theta r}=m_{z r}=0 \quad \text { on } r=a ; \\
\bar{q}_{r}=0 \quad \text { on } r=a,
\end{gathered}
$$

where the definition of $\bar{q}_{r}$ can be found in Fleck et al. (1994). An alternative boundary condition instead of equation (33a) is:

$$
m_{r r}=0 \quad \text { on } r=a .
$$

On the end faces there are the following boundary conditions:

$$
\begin{aligned}
& u_{r}=0, \quad u_{\theta}=0, \quad u_{z}=0 \quad \text { on } z=0 ; \\
& u_{r}=0, \quad u_{\theta}=\kappa r L, \quad u_{z}=0 \quad \text { on } z=L ; \\
& m_{r z}=m_{\theta z}=0, \quad m_{z z}^{\prime}=\frac{2}{3} l_{c s}^{2} \Sigma_{0} \kappa^{n}\left(\frac{r^{2}}{3}+l_{c s}^{2}\right)^{\frac{n-1}{2}} \quad \text { on } z=0 ; \\
& m_{r z}=m_{\theta z}=0, \quad m_{z z}^{\prime}=\frac{2}{3} l_{c s}^{2} \Sigma_{0} \kappa^{n}\left(\frac{r^{2}}{3}+l_{c s}^{2}\right)^{\frac{n-1}{2}} \quad \text { on } z=L,
\end{aligned}
$$

where $L$ is the total length of the thin wire as shown in figure 1 .

\section{1. $K_{1} \rightarrow \infty$}

If the solid is assumed to be incompressible for bend-torsion, thus $K_{1} \rightarrow \infty$, we can find that the corresponding solutions to the above boundary value problem is:

$$
\begin{aligned}
& u_{1}=-\kappa x_{2} x_{3}, \quad u_{2}=\kappa x_{1} x_{3}, \quad u_{3}=0 ; \\
& \omega_{r}=-\frac{1}{2} \kappa r, \quad \omega_{\theta}=0, \quad \omega_{z}=\kappa z .
\end{aligned}
$$

It is verified as following:

From the displacement field, we have the non-vanishing components of strain:

$$
\varepsilon_{13}^{\prime}=\varepsilon_{31}^{\prime}=-\frac{1}{2} \kappa x_{2}, \quad \varepsilon_{23}^{\prime}=\varepsilon_{32}^{\prime}=\frac{1}{2} \kappa x_{1}, \quad \varepsilon_{e}=\frac{1}{\sqrt{3}} \kappa r
$$

and the non-vanishing deviatoric components of curvature tensor are:

$$
\chi_{11}^{\prime}=\chi_{22}^{\prime}=-\frac{1}{2} \kappa, \quad \chi_{33}^{\prime}=\kappa, \quad \chi_{e}=\kappa .
$$


The spherical part of curvature tensor is:

$$
\chi_{m}=\frac{1}{3} \chi_{k k}=0
$$

Here, the simple power law relationship between $\Sigma_{e}$ and $E_{e}$ using in Fleck et al. (1994) is adopted also:

$$
\Sigma_{e}=\Sigma_{0} E_{e}^{n}
$$

The non-vanishing component of Cauchy stress only is:

$$
\sigma_{\theta z}=s_{\theta z}=\frac{1}{3} \Sigma_{0} \kappa E_{e}^{n-1} r
$$

The non-vanishing deviatoric components of couple stress are:

$$
m_{r r}^{\prime}=-\frac{1}{3} l_{c s}^{2} \Sigma_{0} \kappa E_{e}^{n-1}, \quad m_{\theta \theta}^{\prime}=-\frac{1}{3} l_{c s}^{2} \Sigma_{0} \kappa E_{e}^{n-1}, \quad m_{z z}^{\prime}=\frac{2}{3} l_{c s}^{2} \Sigma_{0} \kappa E_{e}^{n-1}
$$

where

$$
E_{e}=\kappa\left(\frac{r^{2}}{3}+l_{c s}^{2}\right)^{1 / 2}
$$

From equation (44), we find that the non-vanishing component of Cauchy stress is only related with $r$, the equilibrium equations of stress, i.e., equation (28) are met.

Since the components of couple stress in (45) are only related with $r,(29)$ can be reduced:

$$
\begin{aligned}
& \frac{\partial m_{r r}}{\partial r}+\frac{m_{r r}-m_{\theta \theta}}{r}=0, \\
& \frac{1}{r} \frac{\partial m_{m}}{\partial \theta}=0, \\
& \frac{\partial m_{m}}{\partial z}=0
\end{aligned}
$$

$m_{m}$ can not be determined from (31) and becomes an independent unknown variable. Combining the boundary condition, equation (33b), we obtain:

$$
m_{m}=\frac{1}{3} l_{c s}^{2} \kappa^{n} \Sigma_{0}\left(\frac{1}{3} r^{2}+l_{c s}^{2}\right)^{\frac{n-1}{2}} .
$$

The torque produced by the spherical part of moment in the present theory is:

$$
Q_{1}=\int_{0}^{2 \pi} \int_{0}^{a} m_{m} r \mathrm{~d} r \mathrm{~d} \theta=\frac{2 \pi l_{c s}^{2} \Sigma_{0} \kappa^{n}}{n+1}\left[\left(\frac{a^{2}}{3}+l_{c s}^{2}\right)^{\frac{n+1}{2}}-l_{c s}^{n+1}\right] .
$$

The overall torque produced by $\sigma_{\theta z}, m_{z z}^{\prime}, m_{m}$ on the end face is:

$$
Q=\frac{6 \pi \Sigma_{0} \kappa^{n}}{n+3}\left[\left(\frac{a^{2}}{3}+l_{c s}^{2}\right)^{\frac{n+3}{2}}-l_{c s}^{n+3}\right]
$$




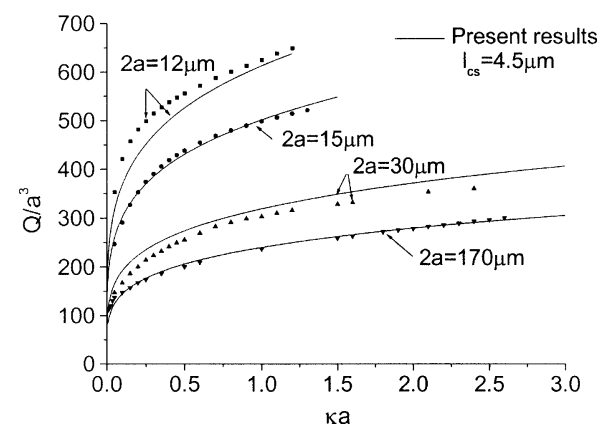

Figure 2. Plots of torque against the surface strain for copper wires with different diameters and $K_{1} \rightarrow \infty$. The solid lines denote the theoretical results in the present paper and the dotted lines denote the experiment results (Fleck et al., 1994).

The above equation is the same as equation (6.9) in Fleck et al. (1994). From simulating the tensile curves of experiment we take $n=0.22$. If we choose a torsional response curve of $2 a=15 \mu \mathrm{m}$ as a calibration curve, we get $l_{c s}=4.5 \mu \mathrm{m}$. Comparisons with the experimenta results are shown in figure 2 .

From above we proved that $\omega_{i}=\theta_{i}$ is the corresponding solution to the special boundary conditions of equations (32)-(37). If we take another set of boundary conditions, for example:

$$
\begin{aligned}
& u_{r}=0, \quad u_{\theta}=0, \quad u_{z}=0 \quad \text { on } z=0 ; \\
& u_{r}=0, \quad u_{\theta}=\kappa r L, \quad u_{z}=0 \quad \text { on } z=L ; \\
& m_{r z}=m_{\theta z}=0, \quad \omega_{z}=0 \quad \text { on } z=0 ; \\
& m_{r z}=m_{\theta z}=0, \quad \omega_{z}=0 \quad \text { on } z=L ; \\
& m_{\theta r}=m_{z r}=0 \quad \text { on } r=a ; \\
& m_{r r}=0 \quad \text { on } r=a .
\end{aligned}
$$

One can find another set of solution $\omega_{i} \neq \theta_{i}$ to the above set of boundary conditions and here it is omitted.

\section{2. $K_{1}$ has a limit value}

If $K_{1}$ has a limit value, one will know also that $\omega_{i}=\theta_{i}$ is no longer a solution to the boundary conditions of equations (32)-(37) and $\alpha_{i j} \neq 0$. The anti-symmetric stress $\tau_{i j}$ will not vanish.

We can prove that the displacement field in (38) is the correct displacement solution. From the displacement field the material rotation field can be obtained:

$$
\theta_{r}=-\frac{1}{2} \kappa r, \quad \theta_{\theta}=0, \quad \theta_{z}=\kappa z .
$$

The non-vanishing components of strain tensor and the effective strain are:

$$
\varepsilon_{\theta z}=\varepsilon_{z \theta}=\frac{1}{2} \kappa r, \quad \varepsilon_{e}^{2}=\frac{\kappa^{2} r^{2}}{3} .
$$

We assume that $\chi_{i j}$ is only related to $r$ and $\omega_{\theta}=\theta_{\theta}, \omega_{z}=\theta_{z}$, then one can obtain the following special solutions for the boundary value problem:

$$
\omega_{r}=-\frac{1}{2} \kappa r+\tilde{\omega}_{r}(r), \quad \omega_{\theta}=0, \quad \omega_{z}=\kappa z .
$$


Now $\tilde{\omega}_{r}(r)$ is to be determined as following:

From (59), the non-vanishing deviatoric parts of curvature tensor are:

$$
\begin{aligned}
& \chi_{r r}^{\prime}=-\frac{1}{2} \kappa+\frac{2}{3} \tilde{\omega}_{r}^{\prime}-\frac{1}{3} \frac{\tilde{\omega}_{r}}{r}, \\
& \chi_{\theta \theta}^{\prime}=-\frac{1}{2} \kappa+\frac{2}{3} \frac{\tilde{\omega}_{r}}{r}-\frac{1}{3} \tilde{\omega}_{r}^{\prime}, \\
& \chi_{z z}^{\prime}=\kappa-\frac{1}{3} \frac{\tilde{\omega}_{r}}{r}-\frac{1}{3} \tilde{\omega}_{r}^{\prime},
\end{aligned}
$$

and the effective rotation gradient is:

$$
\chi_{e}^{2}=\frac{2}{3}\left[\left(\chi_{r r}^{\prime}\right)^{2}+\left(\chi_{\theta \theta}^{\prime}\right)^{2}+\left(\chi_{z z}^{\prime}\right)^{2}\right]
$$

then

$$
E_{e}^{2}=\frac{\kappa^{2} r^{2}}{3}+\frac{2}{3} l_{c s}^{2}\left[\left(-\frac{\kappa}{2}+\frac{2}{3} \tilde{\omega}_{r}^{\prime}-\frac{1}{3} \frac{\tilde{\omega}_{r}}{r}\right)^{2}+\left(-\frac{\kappa}{2}+\frac{2}{3} \frac{\tilde{\omega}_{r}}{r}-\frac{1}{3} \tilde{\omega}_{r}^{\prime}\right)^{2}+\left(\kappa-\frac{1}{3} \tilde{\omega}_{r}^{\prime}-\frac{1}{3} \frac{\tilde{\omega}_{r}}{r}\right)^{2}\right]
$$

The non-vanishing anti-symmetric stresses are:

$$
\tau_{\theta z}=-\tau_{z \theta}=2 C_{1} \tilde{\omega}_{r}
$$

The non-vanishing components of Cauchy stress are:

$$
t_{\theta z}=\frac{\Sigma_{0} \kappa}{3} E_{e}^{n-1} r+2 C_{1} \tilde{\omega}_{r}, \quad t_{z \theta}=\frac{\Sigma_{0} \kappa}{3} E_{e}^{n-1} r-2 C_{1} \tilde{\omega}_{r} .
$$

The non-vanishing components of couple stress are:

$$
\begin{aligned}
& m_{r r}=m_{r r}^{\prime}+m_{m}=\frac{2}{3} \Sigma_{0} E_{e}^{n-1} l_{c s}^{2}\left(-\frac{\kappa}{2}+\frac{2}{3} \tilde{\omega}_{r}^{\prime}-\frac{1}{3} \frac{\tilde{\omega}_{r}}{r}\right)+\frac{K_{1} l_{c s}^{2}}{3}\left(\tilde{\omega}_{r}^{\prime}+\frac{\tilde{\omega}_{r}}{r}\right), \\
& m_{\theta \theta}=m_{\theta \theta}^{\prime}+m_{m}=\frac{2}{3} \Sigma_{0} E_{e}^{n-1} l_{c s}^{2}\left(-\frac{\kappa}{2}+\frac{2}{3} \frac{\tilde{\omega}_{r}}{r}-\frac{1}{3} \tilde{\omega}_{r}^{\prime}\right)+\frac{K_{1} l_{c s}^{2}}{3}\left(\tilde{\omega}_{r}^{\prime}+\frac{\tilde{\omega}_{r}}{r}\right), \\
& m_{z z}=m_{z z}^{\prime}+m_{m}=\frac{2}{3} \Sigma_{0} E_{e}^{n-1} l_{c s}^{2}\left(\kappa-\frac{1}{3} \tilde{\omega}_{r}^{\prime}-\frac{1}{3} \frac{\tilde{\omega}_{r}}{r}\right)+\frac{K_{1} l_{c s}^{2}}{3}\left(\tilde{\omega}_{r}^{\prime}+\frac{\tilde{\omega}_{r}}{r}\right) .
\end{aligned}
$$

Now, from equation (64) we can find that the non-vanishing components are only related to $r$, so equation (28) is satisfied.

Substituting equations (63) and (65) into (29), one can find that the second and third equations in (29) are met automatically and from the first equation in (29) we can obtain:

$$
\begin{gathered}
2(n-1) E_{e}^{n-2} l_{c s}^{2} \Sigma_{0}\left(-\frac{\kappa}{2}+\frac{2}{3} \tilde{\omega}_{r}^{\prime}-\frac{1}{3} \frac{\tilde{\omega}_{r}}{r}\right) E_{e}^{\prime}+2 E_{e}^{n-1} l_{c s}^{2} \Sigma_{0}\left(\frac{2}{3} \tilde{\omega}_{r}^{\prime \prime}-\frac{1}{3} \frac{\tilde{\omega}_{r}^{\prime}}{r}+\frac{1}{3} \frac{\tilde{\omega}_{r}}{r^{2}}\right) \\
+2 E_{e}^{n-1} l_{c s}^{2} \Sigma_{0}\left(\tilde{\omega}_{r}^{\prime} r^{-1}-\tilde{\omega}_{r} r^{-2}\right)+K_{1} l_{c s}^{2}\left(\tilde{\omega}_{r}^{\prime \prime}+\tilde{\omega}_{r}^{\prime} r^{-1}-\tilde{\omega}_{r} r^{-2}\right)=12 C_{1} \tilde{\omega}_{r},
\end{gathered}
$$


and $\tilde{\omega}_{r}$ can be solved from the above equation through numerical calculation and it must meet the following conditions:

$$
\left.\tilde{\omega}_{r}\right|_{r=0}=0,\left.\quad m_{r r}\right|_{r=a}=0 .
$$

The corresponding overall torque produced by $t_{\theta z}$ and $m_{z z}$ can be expressed as:

$$
Q=\int_{0}^{2 \pi} \int_{0}^{a} t_{\theta z} r^{2} \mathrm{~d} r \mathrm{~d} \theta+\int_{0}^{2 \pi} \int_{0}^{a} m_{z z} r \mathrm{~d} r \mathrm{~d} \theta .
$$

The numerical results are shown in figures 3 and 4 for a set of parameters, $\Sigma_{0}=237 \mathrm{MPa}, n=0.22$, $l_{c s}=4.5 \mu \mathrm{m}, 2 a=15 \mu \mathrm{m}, \kappa=1 / 15, \Sigma_{0} / K_{1}=0.01, C_{1} / K_{1}=0.1$. From figures 3 and 4 we find that the

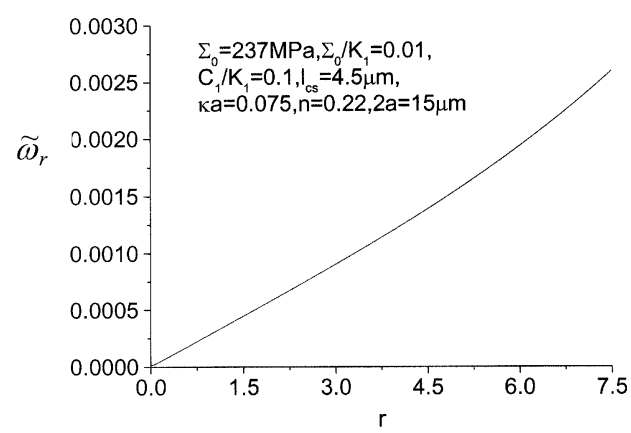

Figure 3. Relation curve of $r \sim \tilde{\omega}_{r}$ for $\Sigma_{0}=237 \mathrm{MPa}, \Sigma_{0} / K_{1}=0.01, C_{1} / K_{1}=0.1$.

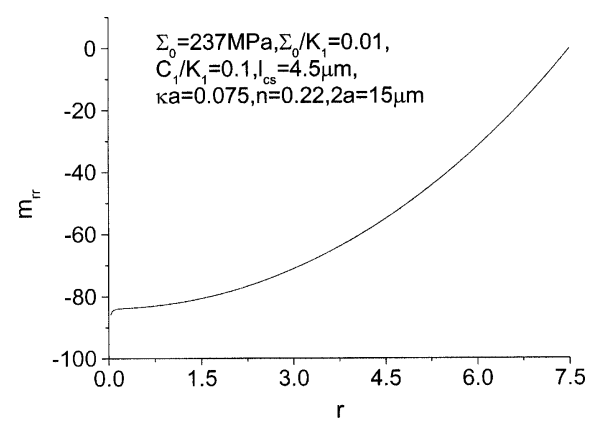

Figure 4. Relation curve of $r \sim m_{r r}$ for $\Sigma_{0}=237 \mathrm{MPa}, \Sigma_{0} / K_{1}=0.01, C_{1} / K_{1}=0.1$.

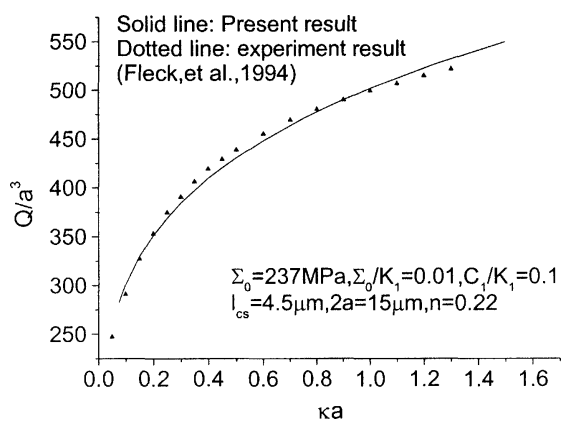

Figure 5. Plot of torque against the surface strain for copper wire with diameter $2 a=15 \mu \mathrm{m}$ and $K_{1}$ is a finite value. The solid line denotes the theoretical result and the dotted line denotes the experiment result (Fleck et al., 1994). 
boundary conditions in (67) are met and for this kind of case one can find that $\omega_{i} \neq \theta_{i}$. The curve of $\kappa a$ versus $Q / a^{3}$ for $2 a=15 \mu \mathrm{m}$ is shown in figure 5. From figure 5 one can find that the present results are quite consistent with the experiments results. Comparing with the theoretical curve of $2 a=15 \mu \mathrm{m}$ in figure 2, one can find that the finite value of $K_{1}$ has little influence on the overall torque. It means that if $K_{1}$ is large enough compared with $\Sigma_{0}$, the predictions based on $K_{1} \rightarrow \infty$ are correct.

\section{Ultra-thin beam bend}

Stolken and Evans (1998) did the bend experiment and observed a strong effect whereby thin beams display much stronger plastic work hardening than thick ones and no size dependence was observed in the tension test.

In this section ultra-thin beam bending with different thickness is analyzed using the theory in the present paper. Cartesian $\left(x_{1}, x_{2}, x_{3}\right)$ coordinates are adopted as shown in figure $6 . \kappa$ is the curvature, $h$ is the beam's thickness and $b$ is the beam's width.

The classical displacement fields are:

$$
u_{1}=\kappa x_{1} x_{2}, \quad u_{2}=-\kappa\left(x_{1}^{2}+x_{2}^{2}\right) / 2, \quad u_{3}=0 .
$$

The non-vanishing strain components are:

$$
\varepsilon_{11}=-\varepsilon_{22}=\kappa x_{2} .
$$

The components of the overall rotation vector are:

$$
\theta_{1}=0, \quad \theta_{2}=0, \quad \theta_{3}=-\kappa x_{1} .
$$

From simulating the tensile test results of thin beams, the relation between the stress and plastic strain can be expressed as (Stolken and Evans, 1998):

$$
\sigma=\Sigma_{0}+\varepsilon_{p l} E_{p},
$$

where $\Sigma_{0}$ is the yield strength, $\varepsilon_{p l}$ is the plastic strain and $E_{p}$ the hardening coefficient.

The hardening relation including the effect of strain gradient plasticity is:

$$
\Sigma_{e}=\frac{\sqrt{3}}{2} \Sigma_{0}+\frac{3}{4} E_{p} E_{e}, \quad E_{e}=\sqrt{\varepsilon_{e}^{2}+l_{c s}^{2} \chi_{e}^{2}} .
$$

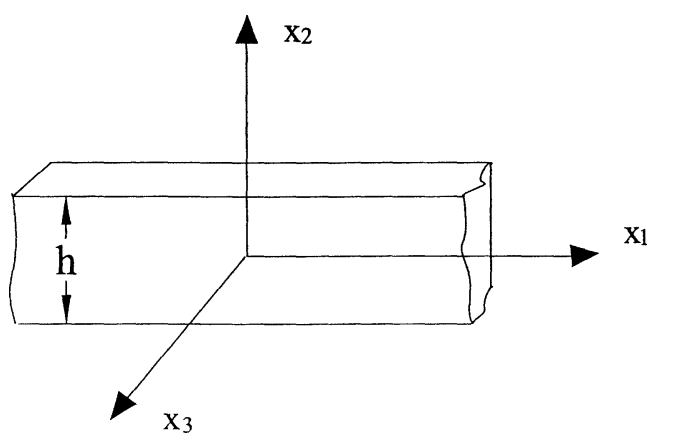

Figure 6. Coordinate system $\left(x_{1}, x_{2}, x_{3}\right)$ on an ultra-thin beam. 
The non-vanishing components of Cauchy stress and the moment in Stolken and Evans (1998) are:

$$
\sigma_{11}=\frac{4 \Sigma_{e}}{3 E_{e}} \kappa x_{2}, \quad \sigma_{33}=\frac{2 \Sigma_{e}}{3 E_{e}} \kappa x_{2}, \quad m_{31}=-\frac{2 \Sigma_{e}}{3 E_{e}} l_{c s}^{2} \kappa
$$

and

$$
\tau_{i j}=0 \quad(i, j=1,2,3) .
$$

The boundary conditions on the lateral faces are:

$$
\begin{aligned}
& \sigma_{12}=\sigma_{22}=\sigma_{32}=0, \quad m_{12}=m_{22}=m_{32}=0 \quad \text { on } x_{2}= \pm h / 2 ; \\
& \sigma_{13}=\sigma_{23}=0, \quad m_{13}=m_{23}=m_{33}=0 \quad \text { on } x_{3}= \pm b / 2 ; \\
& \sigma_{33}=\frac{2 \Sigma_{e}}{3 E_{e}} \kappa x_{2} \quad \text { on } x_{3}= \pm b / 2 .
\end{aligned}
$$

The boundary conditions on the end faces are:

$$
\begin{array}{ll}
\sigma_{11}=\frac{4 \Sigma_{e}}{3 E_{e}} \kappa x_{2}, & t_{21}=t_{31}=0, \quad \text { on } x_{1}= \pm L / 2 ; \\
m_{11}=m_{21}=0, & m_{31}=-\frac{2 \Sigma_{e}}{3 E_{e}} l_{c s}^{2} \kappa
\end{array}
$$

where $E_{e}=\sqrt{\frac{4}{3} \kappa^{2} x_{2}^{2}+\frac{2}{3} \kappa^{2}}$.

Since $\tau_{i j}$ specially vanishes for the ultra-thin beam bend with the theory proposed by Fleck and Hutchinson (1993), we can easily find that while using the present strain gradient theory to investigate the same problem with the same boundary conditions and same hardening law as Stolken and Evans (1998), the solutions to this special boundary conditions are as following:

$$
\begin{aligned}
& u_{1}=\kappa x_{1} x_{2}, \quad u_{2}=-\kappa\left(x_{1}^{2}+x_{2}^{2}\right) / 2, \quad u_{3}=0 ; \\
& \omega_{1}=0, \quad \omega_{2}=0, \quad \omega_{3}=-\kappa x_{1} .
\end{aligned}
$$

The equilibrium equations of equations (28)-(30) are all met.

It must be noted that if the boundary conditions change the solution will change also, i.e., $\omega_{i} \neq \theta_{i}$.

\section{A new hardening law}

In the above, the hardening law proposed by Fleck and Hutchinson (1993) is used to analyze the two examples. Now, another new hardening relation, in which not only the rotation gradient but also the stretch gradient is considered, is proposed as following. Since the stretch gradient is considered and it is the second differential of displacement, in order to avoid a higher order differential of displacement emerging in the equilibrium equations, the new hardening law is in the incremental form and at each incremental step $\eta_{1}$ is only a parameter to influence the tangent modulus. The concrete hardening law is expressed as following:

$$
\dot{\Sigma}_{e}=f^{\prime}\left(E_{e}\right)\left(1+\frac{l_{1} \eta_{1}}{E_{e}}\right)^{1 / 2} \dot{E}_{e},
$$

$\eta_{1}$ in the above equation denotes the effective stretching gradient and can be expressed as:

$$
\eta_{1}=\sqrt{\eta_{i j k}^{(1)} \eta_{i j k}^{(1)}}
$$


where $\eta_{i j k}^{(1)}$ denotes the stretching gradient tensor and can be obtained from the second differential of the displacement vector, which can be found in Chen and Wang (2000). $l_{1}$ is the length scale related with the stretch gradient.

The corresponding constitutive relations in the incremental form are as follows:

$$
\begin{aligned}
& \dot{s}_{i j}=\frac{2 \dot{\Sigma}_{e}}{3 E_{e}} \varepsilon_{i j}^{\prime}-\frac{2 \Sigma_{e}}{3 E_{e}^{2}} \varepsilon_{i j}^{\prime} \dot{E}_{e}+\frac{2 \Sigma_{e}}{3 E_{e}} \dot{\varepsilon}_{i j}^{\prime} ; \\
& \dot{m}_{i j}^{\prime}=\frac{2}{3} l_{c s}^{2} \frac{\dot{\Sigma}_{e}}{E_{e}} \chi_{i j}^{\prime}-\frac{2 l_{c s}^{2} \Sigma_{e}}{3 E_{e}^{2}} \chi_{i j}^{\prime} \dot{E}_{e}+\frac{2 l_{c s}^{2} \Sigma_{e}}{3 E_{e}} \dot{\chi}_{i j}^{\prime} ; \\
& \dot{\sigma}_{m}=\frac{1}{3} \dot{\sigma}_{k k}, \quad \dot{m}_{m}=\frac{1}{3} \dot{m}_{k k} .
\end{aligned}
$$

\subsection{Thin wire torsion}

In this section the new hardening law, equation (83), is adopted to analyze the torsion of thin wires with different diameters. From the above section we know that equations (38) and (39) are the true field for the special boundary conditions (32)-(37). Here we take (38) and (39) to check the overall torque produced by the new hardening law.

The anti-symmetric stress vanishes. The non-vanishing components of strain and the deviatoric components of curvature tensor are the same as equations (40)-(41).

From the displacement field, the stretching gradient can be obtained:

$$
\eta_{i j k}^{(1)} \eta_{i j k}^{(1)}=0
$$

then

$$
\eta_{1}=0
$$

Substituting the effective strain, effective rotation gradient and the effective stretching gradient into the hardening law, equation (83), then:

$$
\dot{\Sigma}_{e}=n \Sigma_{0} E_{e}^{n-1} \dot{E}_{e}
$$

After integrating (90) one can obtain the following equation:

$$
\Sigma_{e}=\Sigma_{0} E_{e}^{n}
$$

Thus, it is reasonable and convenient to use (91) to solve the problem of thin wire torsion and from above Section 4.1 , we can easily obtain the overall torque.

\subsection{Ultra-thin beam bending}

In this section the new hardening law, equation (83), is adopted to analyze bending of ultra-thin beams with different thickness. From above, we know that equations (81) and (82) are the true field for the special boundary conditions (76)-(80). Here we take equations (81) and (82) to check the overall moment produced by the new hardening law. 
The anti-symmetric stress vanishes. The non-vanishing components of strain are the same as equation (70) and the deviatoric components of curvature tensor are:

$$
\chi_{31}=-\kappa, \quad \chi_{e}=\sqrt{\frac{2}{3}} \kappa .
$$

From the displacement field, the stretching gradient can be obtained:

$$
\eta_{i j k}^{(1)} \eta_{i j k}^{(1)}=\frac{76}{75} \kappa^{2}
$$

Substituting the effective strain and effective strain gradient into the hardening law, equation (83), then:

$$
\dot{\Sigma}_{e}=\frac{3}{4} E_{p}\left[1+\frac{\sqrt{38} l_{1}}{5\left(2 x_{2}^{2}+l_{c s}^{2}\right)^{1 / 2}}\right]^{1 / 2} \dot{E}_{e} .
$$

From (94), we find that the term reflecting the effect of strain gradient has no relation with the deformation history, so for the problem of ultra-thin beam bend, after integrating (94) one obtains the following equation:

$$
\Sigma_{e}=\left(\frac{\sqrt{3}}{2} \Sigma_{0}+\frac{3}{4} E_{p} E_{e}\right)\left[1+\frac{\sqrt{38} l_{1}}{5\left(2 x_{2}^{2}+l_{c s}^{2}\right)^{1 / 2}}\right]^{1 / 2} .
$$

Thus, it is reasonable and convenient to use (95) to solve the problem. Here the overall moments at the end faces are taken into account:

The non-vanishing stress components and couple stress components at the end faces are:

$$
\begin{aligned}
& \sigma_{11}=2 S_{11}=\frac{4 \Sigma_{e}}{3 E_{e}} \varepsilon_{11}^{\prime}=\left[\frac{\Sigma_{0}}{\sqrt{2}}\left(l_{c s}^{2}+2 x_{2}^{2}\right)^{-1 / 2}+\kappa E_{p}\right]\left[1+\frac{\sqrt{38} l_{1}}{5\left(2 x_{2}^{2}+l_{c s}^{2}\right)^{1 / 2}}\right]^{1 / 2} x_{2} ; \\
& m_{31}=\frac{2 \Sigma_{e}}{3 E_{e}} l_{c s}^{2} \chi_{31}=\left[\frac{-l_{c s}^{2} \Sigma_{0}}{\sqrt{2}}\left(l_{c s}^{2}+2 x_{2}^{2}\right)^{-1 / 2}-\frac{l_{c s}^{2} \kappa}{2} E_{p}\right]\left[1+\frac{\sqrt{38} l_{1}}{5\left(2 x_{2}^{2}+l_{c s}^{2}\right)^{1 / 2}}\right]^{1 / 2} .
\end{aligned}
$$

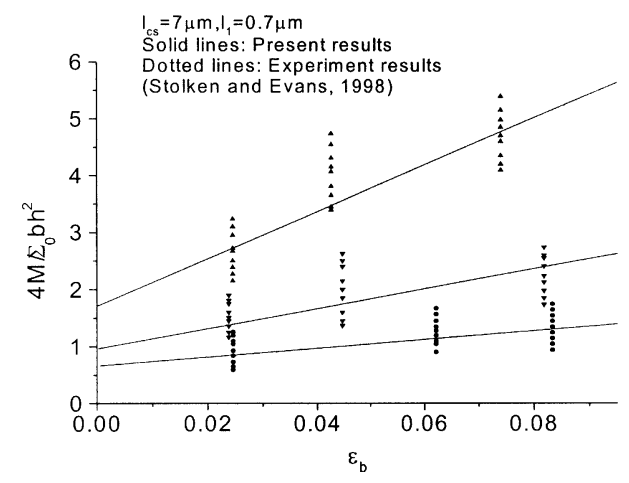

Figure 7. Plots of bending moment against the surface strain for three beams with different thickness. The dotted lines denote the theoretical results by Fleck and Hutchinson (1997) and the solid lines denote the present results, the various symbols denote the experiment results (Stolken and Evans, 1998). 
Then the overall moments produced by the stresses and couple stresses at the end faces are

$$
M=2 \int_{0}^{h / 2} \sigma_{11} b x_{2} \mathrm{~d} x_{2}-2 \int_{0}^{h / 2} m_{31} b \mathrm{~d} x_{2} .
$$

The comparisons of equation (98) with the test results for Ni beam bend with different thickness (Stolken and Evans, 1998) are shown in figure 7, where $l_{1}=0.1 l_{c s}$ similar to Stolken and Evans (1998). From figure 7 we can find that all curves predicted by (98) for different thickness are quite consistent with the test results and here $l_{c s}=7 \mu \mathrm{m}$.

\section{Flow theory}

In this section the $J_{2}$ flow theory version of the present theory for crystalline solids is given briefly following Section 3, in which the strain gradient deformation theory is given.

The strain tensor consists of elastic and plastic parts. The curvature tensor also consists of the two corresponding parts. Thus the strain rate and the curvature tensor rate can be expressed:

$$
\dot{\varepsilon}_{i j}=\dot{\varepsilon}_{i j}^{e}+\dot{\varepsilon}_{i j}^{p}, \quad \dot{\chi}_{i j}^{p}=\dot{\omega}_{i, j} .
$$

Assuming the existence of couple stresses in the elastic-plastic body. The elastic strain state $\varepsilon_{i j}^{e}$ is obtained:

$$
\varepsilon_{i j}^{e}=\mu_{i j k l} \sigma_{k l}
$$

where $\mu_{i j k l}$ is the elastic compliance tensor.

Since the angular displacement $\omega_{i}$ is directly related to the plastic curvature tensor $\chi_{i j}^{p}$, hence we only need to address the constitutive equation for $\chi_{i j}^{p}$.

The yield surface $\Phi$ can be written as:

$$
\Phi=\Phi\left(\sigma_{e}, m_{e}, Y\right)
$$

where $\sigma_{e}=\sqrt{\frac{3}{2} s_{i j} s_{i j}}$ is the Von Mises effective stress, $m_{e}=\sqrt{\frac{3}{2} m_{i j}^{\prime} m_{i j}^{\prime}}$ is the effective couple stress, and $Y$ is the current flow stress.

Plastic flow is normal to the yield surface such that:

$$
\dot{\varepsilon}_{i j}^{p}=\dot{\lambda} \frac{\partial \Phi}{\partial \sigma_{i j}}, \quad l_{c s} \dot{\chi}_{i j}^{p}=l_{c s}^{-1} \dot{\lambda} \frac{\partial \Phi}{\partial m_{i j}} .
$$

According to Fleck and Hutchinson (1993), $\Sigma_{e}$ is called the overall effective stress defined by equation (23) and the overall effective plastic strain rate is defined as $\dot{E}_{e}^{p}=\sqrt{\left(\dot{\varepsilon}_{e}^{p}\right)^{2}+\left(l_{c s} \dot{\chi}_{e}^{p}\right)^{2}}$, where the effective strain rate is $\dot{\varepsilon}_{e}^{p}=\sqrt{\frac{2}{3} \dot{\varepsilon}_{i j}^{p} \dot{\varepsilon}_{i j}^{p}}$ and the effective curvature tensor rate is $\dot{\chi}_{e}^{p}=\sqrt{\frac{2}{3} \dot{\chi}_{i j}^{p} \dot{\chi}_{i j}^{p}}$. Then the yield surface (101) generalizes to:

$$
\Phi\left(\Sigma_{e}, Y\right)=\Sigma_{e}-Y=0 .
$$


The plastic strain rate and the plastic curvature tensor rate can be given

$$
\dot{\varepsilon}_{i j}^{p}=\frac{3}{2 h\left(\Sigma_{e}\right)} \frac{s_{i j}}{\Sigma_{e}} \dot{\Sigma}_{e}, \quad l_{c s} \dot{\chi}_{i j}^{p}=\frac{3}{2 h\left(\Sigma_{e}\right)} \frac{l_{c s}^{-1} m_{i j}^{\prime}}{\Sigma_{e}} \dot{\Sigma}_{e} .
$$

According to the hardening law proposed by Fleck et al. (1994) and Fleck and Hutchinson (1993):

$$
\begin{gathered}
\dot{\Sigma}_{e}=f^{\prime}\left(E_{e}^{p}\right) \dot{E}_{e}^{p}=A\left(E_{e}^{p}\right) \dot{E}_{e}^{p} \\
\dot{\Sigma}_{e}=\frac{3}{2} \frac{s_{i j}}{\Sigma_{e}} \dot{s}_{i j}+\frac{3}{2} \frac{l_{c s}^{-1} m_{i j}^{\prime}}{\Sigma_{e}} l_{c s}^{-1} \dot{m}_{i j}^{\prime} .
\end{gathered}
$$

For this kind of hardening law, the tangent modulus $h$ of the stress $\Sigma_{e}$ versus plastic strain $E_{e}^{p}$ curve in simple tension is $h=\dot{\Sigma}_{e} / \dot{E}_{e}^{p}=A\left(E_{e}^{p}\right)$. While considering the stretching gradient, only the tangent modulus $h$ changes and $h=A\left(E_{e}^{p}\right)\left(1+\frac{l_{1} \eta_{1}}{E_{e}^{p}}\right)^{1 / 2}$.

The constitutive equation for the anti-symmetric stress rate is:

$$
\dot{\tau}_{i j}=2 C_{1} \dot{\alpha}_{i j}=2 C_{1} e_{i j k}\left(\dot{\omega}_{k}-\dot{\theta}_{k}\right)
$$

\section{Conclusions}

(1) The present strain gradient theory for crystalline solid fits within the mathematical framework of the general couple stress theory. But the present theory differs from the reduced couple stress theory. In the present theory, $\omega_{i}$ plays the role of the plastic rotation.

(2) In the new strain gradient theory, $\omega_{i}$ is different from $\theta_{i}$, generally speaking that $\alpha_{i j} \neq 0$. The equilibrium equations for Cauchy stresses and couple stresses are second order differential of displacement $u_{i}$ and angular displacement $\omega_{i}$ respectively and no higher order terms are introduced.

(3) The strain energy density $w$ is assumed to consist of two parts $w_{0}$ and $w_{1}$, the former is a function of the strain tensor $\varepsilon_{i j}$ and the curvature tensor $\chi_{i j}$, the latter is a function of the relative rotation tensor $\alpha_{i j}$, which means that the anti-symmetric stress $\tau_{i j}$ is only the function of the elastic rotation vector and the relative rotation tensor $\alpha_{i j}$ has no effect on $\sigma_{i j}$ and $m_{i j}$.

(4) A simple linear elastic relation between $\alpha_{i j}$ and $\tau_{i j}$ is used to analyze the torsion of thin wires and bending of ultra-thin beams. The elastic modulus is $C_{1}$. While $C_{1}$ equals zero, the Cauchy stress tensor becomes symmetric and the Cauchy stresses and couple stresses in their own equilibrium equations are one to one correspondences.

(5) Since $\omega_{i} \neq \theta_{i}, \chi_{k k} \neq 0$, the volumetric modulus of bend-torsion $K_{1}$ can be naturally introduced and the spherical part of couple stress can be determined according to the boundary conditions.

(6) Using the new strain gradient theory, the moment on the free boundary can be met exactly and the spherical part of the moment can be obtained.

(7) The rotation, $\omega_{i}$, is treated as an independent kinematic quantity with no direct dependence upon $u_{i}$, this enables the use of $C_{0}$ continuous elements in a finite element formulation, which will give great benefit to the finite element calculation to avoid dramatic sensitivity to the element formulation.

(8) A new hardening law is introduced in an incremental form, in which not only the rotation gradient but also the stretch gradient is considered. The equilibrium equations are still second-order differentials of 
displacement and angular displacement. The effective stretching gradient $\eta_{1}$ is only a parameter to influence the tangent modulus. Using the new hardening law to analyze torsion of thin wires and bending of ultra-thin beams, the analytical results are fairly consistent with the experiment results.

\section{Acknowledgments}

This work is supported by the National Natural Science Foundation of China (No. 19704100), National Science Foundation of Chinese Academy of Sciences (Project KJ951-1-20), CAS K.C. Wong Post-doctoral Research Award Fund and the Post-doctoral Science Fund of China.

\section{References}

Acharya, A., Bassani, J.L., 1995. On non-local flow theories that preserve the classical structure of incremental boundary value problems, in: Micromechanics of Plasticity and Damage of Multiphase Materials, IUTAM Symposium, Paris, Aug. 29-Sept. 1.

Aifantis, E.C., 1984. On the microstructural origin of certain inelastic models. J Eng. Mater-T. ASME 106, 326-330.

Chen, S.H., Wang, T.C., 2000. A new hardening law for strain gradient plasticity. Acta Materialia 48 (16), 3997-4005.

Dillon, O.W., Kratochvil, J.R., Kratochvil, J., 1970. A strain gradient theory of plasticity. Int. J. Solids Structures 6, 1513-1533.

Ebeling, R., Ashby, M.F., 1966. Dispersion hardening of copper single crystals. Phil. Mag. 13, 805-834.

Eringen, A.C., 1968. Theory of micropolar elasticity, in: Leibowitz, H. (Ed.), Fracture, An Advanced Treatise. Academic Press, New York, pp. 621729.

Fleck, N.A., Hutchinson, J.W., 1993. A phenomenological theory for strain gradient effects in plasticity. J. Mech. Phys. Solids 41, $1825-1857$.

Fleck, N.A., Muller, G.M., Ashby, M.F, Hutchinson, J.W., 1994. Strain gradient plasticity: theory and experiment. Acta Metal. Mater. 42 , $475-487$.

Fleck, N.A., Hutchinson, J.W., 1997, in: Hutchinson, J.W., Wu, T.Y. (Eds.), Strain Gradient Plasticity, Advances in Applied Mechanics, Vol. 33. Academic Press, New York, pp. 295-361.

Gao, H., Huang, Y., Nix, W.D., Hutchinson, J.W., 1999. Mechanism-based strain gradient plasticity - I. Theory. J. Mech. Phys. Solids 47, $1239-1263$.

Green, A.E., Mcinnis, B.C., Naghdi, P.M., 1968. Elastic-plastic continua with simple force dipole. Int. J. Engng Sci. 6, $373-394$.

Kelly, D., Nicholson, R.B., 1963. Precipitation hardening. Prog. Mater. Sci. 10 (3), 149-391.

Lloyd, D.J., 1994. Particle reinforced aluminum and magnesium matrix composites. Int. Mater. Rev. 39, 1-23.

Ma, Q., Clarke, D.R., 1995. Size dependent hardness in silver single crystals. J. Mater. Res. 10, 853-863.

McElhaney, K.W., Vlassak, J.J., Nix, W.D., 1998. Determination of indenter tip geometry and indentation contact area for depth-sensing indentation experiments. J. Mater. Res. 13, 1300-1306.

Mindlin, R.D., 1963. Influence of couple-stress on stress concentrations. Exp. Mech. 3, 1-7.

Mindlin, R.D., 1964. Microstructure in linear elasticity. Arch. Rational Mech. Anal. 16, 51-78.

Muhlhaus, H.B., Aifantis, E.C., 1991. The influence of microstructure-induced gradients on the localization of deformation in viscoplastic materials. Acta Mechanica 89, 217-231.

Naghdi, P.M., Srinivasa, A.R., 1993-a. A dynamical theory of structured solids. I basic developments. Phil. Trans. R. Soc. Lond. A 345 , $425-458$.

Naghdi, P.M., Srinivasa, A.R., 1993-b. A dynamical theory of structured solids. II special constitutive equations and special cases of the theory. Phil.

Trans. R. Soc. Lond. A 345, 459-476.

Naghdi, P.M., Srinivasa, A.R., 1994. Some general results in the theory of crystallographic slip. ZAMP 45, 687-732.

Nix, W.D., 1989. Mechanical properties of tin films. Metall. Trans. A 20, 2217-2245.

Poole, W.J., Ashby, M.F., Fleck, N.A., 1996. The role of strain gradients in grain size effect for polycrystals. J. Mech. Phys. Solids 44, $465-495$.

Schaefer, H., 1967. Das Cosserat-Kontinuum. Z. Anew. Math. Mech. 47, 485-498.

Shizawa, K., Zbib, H.M., 1999. A thermodynamical theory of gradient elastoplasticity with dislocation density tensor I: Fundamentals. Int. J. Plasticity 15, 899-938.

Smyshlyaev, V.P., Fleck, N.A., 1996. The role of strain gradients in the grain size effect for polycrystals. J. Mech. Phys. Solids 44, $465-495$.

Spencer, A.J.M., 1971. Theory of invariants, in: Eringen, C. (Ed.), Continuum Physics. Academic Press, pp. 239-353.

Stolken, J.S., Evans, A.G., 1998. A microbend test method for measuring the plasticity length scale. Acta Mater. 46, 5109-5115.

Toupin, R., 1962. Elastic materials with couple-stresses. Arch. Rational Mech. Anal. 11, 385-414. 